University of Nebraska - Lincoln

DigitalCommons@University of Nebraska - Lincoln

1988

\title{
Hydrocarbon-Based Discrimination of Three North American Blattella Cockroach Species (Orthoptera: Blattellidae) Using Gas Chromatography
}

D. A. Carlson

USDA-ARS, Insects Affecting Man and Animals Research Laboratory, Gainesville, Florida 32604

R. J. Brenner

USDA-ARS, Insects Affecting Man and Animals Research Laboratory, Gainesville, Florida 32604

Follow this and additional works at: https://digitalcommons.unl.edu/entomologyother

Part of the Entomology Commons

Carlson, D. A. and Brenner, R. J., "Hydrocarbon-Based Discrimination of Three North American Blattella Cockroach Species (Orthoptera: Blattellidae) Using Gas Chromatography" (1988). Entomology Papers from Other Sources. 86.

https://digitalcommons.unl.edu/entomologyother/86

This Article is brought to you for free and open access by the Entomology Collections, Miscellaneous at DigitalCommons@University of Nebraska - Lincoln. It has been accepted for inclusion in Entomology Papers from Other Sources by an authorized administrator of DigitalCommons@University of Nebraska - Lincoln. 


\title{
Hydrocarbon-Based Discrimination of Three North American Blattella Cockroach Species (Orthoptera: Blattellidae) Using Gas Chromatography
}

\author{
D. A. CARLSON AND R. J. BRENNER \\ USDA-ARS, Insects Affecting Man and Animals Research Laboratory, \\ Gainesville, Florida 32604
}

\begin{abstract}
Ann. Entomol. Soc. Am. 81(5): 711-723 (1988)
ABSTRACT The identification of various life stages of three species of cockroaches was accomplished by using gas chromatography for the quantitative determination of cuticular hydrocarbon components. Dried and frozen specimens of wild and laboratory samples of the closely related species, Blattella asahinai Mizukubo and B. germanica (L.), were identified by inspection of chromatographic data. The cuticular hydrocarbons of each species were identified by mass spectrometry and found to be quite unlike those of the distantly related B. vaga Hebard. A statistical technique is described that provides a quantitative approach to numerical pattern recognition of chromatograms for identifying Asian and German cockroaches. In an experiment conducted to evaluate the operational efficiency of this technique for species identification, 63 blind samples of whole cockroaches (37), oöthecae (6), and legs, wings, or cast skins (20) from German-like cockroaches including hybrids, were successfully identified.
\end{abstract}

KEY WORDS Insecta, Asian, nymphs, pattern recognition

RECOGNITION that the Asian cockroach, Blattella asahinai Mizukubo, had become established in 900 $\mathrm{km}^{2}$ of suburban and rural areas of central Florida was complicated initially by the difficulty of identifying these insects that were new to the western hemisphere (Brenner et al. 1988). The first collections were prompted by reports from near Lakeland, Fla., of large populations of cockroaches that lived outdoors and could fly, but otherwise appeared to be German cockroaches, B. germanica (L.).

Adults were determined to be morphologically identical to $B$. asahinai, a species recently described from Okinawa (Mizukubo 1981) that is synonymous with $B$. beybienkoi Roth and is the species most closely related phylogenetically to $B$. germanica (Roth 1985, 1986). This identification was confirmed after exchange of specimens with the initial describer in Japan. Morphological separation of $B$. germanica and $B$. asahinai is difficult. Characteristics described by Roth (1985) in his revision of worldwide Blattella are based primarily on the shape of the tergal gland and medial longitudinal groove in males; no taxonomic keys are available for nymphal stages (which often constitute $90 \%$ of a population of Blattella spp.) or for the adult females. Identification of males involves careful preparation of the cuticle and requires expertise in the taxonomy of cockroaches.

Mizukubo (1981) studied Japanese populations of these two species; he separates females by the larger hind wings in B. asahinai. Thus, we perceived the need to develop a rapid and reliable method of identifying all life stages of these two species, as well as B. vaga Hebard, the third Blattella species found in North America.

Previously, gas chromatography (GC) of cuticular hydrocarbons was used to identify specimens of morphologically indistinguishable members of species complexes, such as Anopheles gambiae A and B mosquitoes (Carlson \& Service 1980) and populations of Africanized and European honey bees (Carlson \& Bolten 1984). Phylogenetic relationships have been studied recently using chemical characters in other insects including mole crickets (Castner \& Nation 1984), the Drosophila virilis group (Bartelt et al. 1986), and Tribolium spp. (Howard 1987).

Preliminary investigations using GC of extracted hydrocarbons of flying wild cockroaches from Lakeland showed distinct differences from those of a laboratory colony of $B$. germanica. Here we report on chemical methods for the rapid and successful identification of $B$. germanica, B. asahinai, and $B$. vaga, using either subjective comparisons of extracted hydrocarbon patterns obtained by GC, or an objective numerical procedure for "pattern recognition" of chromatograms. The characterizations of GC peaks were confirmed by mass spectral interpretation. This is the first identification of hydrocarbons from B. asahinai and the first for North American and Pacific Basin B. germanica.

\section{Materials and Methods}

Adult and nymphal stages of B. asahinai were collected in many locations between Lakeland and Tampa, Fla. and transported to the laboratory to 
establish colonies. Collections were made outdoors where flight behavior was observed and collection of $B$. germanica was unlikely.

Blattella germanica was collected indoors, but at different locations in northcentral Florida and near Tampa where mixing of species was not expected. Other specimens were obtained from established laboratory colonies, including the Orlando "normal" strain (insecticide-susceptible), and the House of Representatives strain (HRDC; insecticide-resistant).

Specimens of B. vaga Hebard were obtained from a laboratory colony initiated from stock sent from the University of California, Riverside. Hybrids of $B$. asahinai females and $B$. germanica males were obtained from colonized material.

Fresh specimens were frozen and maintained at $0^{\circ} \mathrm{C}$ until analysis. Individual specimens were submerged in $1 \mathrm{ml}$ of $\mathrm{n}$-hexane in small vials for 10 $20 \mathrm{~min}$. Hydrocarbons were separated from the extracted lipids using chromatography on minicolumns containing $1 \mathrm{~cm}$ of silica gel (60-200 mesh, J. T. Baker, Philadelphia, Pa.) packed into disposable glass pipets (Carlson \& Service 1980, Carlson $\&$ Bolten 1984). Separation of unsaturated hydrocarbons was done using silver nitrate impregnated thin-layer plates (Carlson \& Bolten 1984). The hydrocarbons were eluted with $1 \mathrm{ml}$ hexane, concentrated to dryness, and redissolved in $50 \mu$ l of hexane for GC analysis.

Simultaneous analysis of two samples by GC used a dual column Tracor Model 540 with on-column injectors (SGE, OC-2, Austin, Tex.), FID detectors, nonpolar fused silica columns $(30 \mathrm{~m}$ by $0.32 \mathrm{~mm}$ inner diameter (ID), DB-1, J and W, Rancho Cordoba, Calif.), and $\mathrm{H}_{2}$ carrier gas. The column oven was programmed from $25-300^{\circ} \mathrm{C}$ at $20^{\circ} \mathrm{C} / \mathrm{min}$. The data were processed by a Nelson Analytical Model 3000 IBM-AT-based system.

Visual inspections of data system output and numerical evaluations were conducted on data from several nymphal age classes as well as adult sexes for German and Asian cockroaches. Sixty Asian and 60 German cockroaches (HRDC strain) were used including 10 each of small nymphs (approximately $2 \mathrm{~mm}$ ), medium nymphs (approximately $5 \mathrm{~mm}$ ), male and female last-instar nymphs (approximately $8-9 \mathrm{~mm}$ ), and older adults $(4-5 \mathrm{~d}$ ) of each sex. This technique is likely to be used to identify specimens submitted from various areas of the country, including locations treated with insect growth regulators that deform adult stages. Therefore, $30 \mathrm{ad}-$ ditional German cockroaches were included as follows: males and females ( 5 each) from a colony initiated in 1984 from materials collected in Alaska; various adults (10) obtained throughout Florida, Alabama, and Louisiana; and males and females (5 each, Orlando normal strain) treated with the growth regulator hydroprene (Patterson \& Koehler 1985). Only 20 specimens of $B$. vaga were analyzed (5 each of small and large nymphs, males and females), because morphological traits of adults and nymphs are readily discernable from Asian and German cockroaches.

Numerical comparisons of GC data were conducted after assignment of Kovats Retention Indices (KI) to peaks. Briefly, these are four-digit numbers assigned to compounds according to their retention times on a GC column when compared to n-alkane standards. The area under each KI peak was integrated and values were expressed as a percentage of the total hydrocarbon composition per specimen.

Gas chromatography mass spectrometry (GCMS) analyses of hydrocarbons were conducted using a Riber-Nermag Model R10-10C mass spectrometer interfaced with a Hewlett-Packard 5790A Series GC. The column oven was fitted with a $30 \mathrm{~m}$ by $0.23 \mathrm{~mm}$ ID column of DB-1 that was temperatureprogrammed from $40-230^{\circ}$ at $60^{\circ} \mathrm{C} / \mathrm{min}$, then 230 $320^{\circ}$ at $18^{\circ} \mathrm{C} / \mathrm{min}$ for electron impact spectra and chemical ionization (CI) spectra using methane. The methyl alkanes of German and Asian cockroach females are described here in groups by the GC peak in which they eluted. The molecular weights of hydrocarbons were identified by their methane CI spectra in which the base peak was always M-1. The analyses were repeated on different samples using a Hewlett-Packard Model 5988A mass spectrometer interfaced with a Hewlett-Packard 5890 Series GC, with a $15 \mathrm{~m}$ by 0.32 mm ID nonpolar cross-linked column, 90:1 split injection, and oven conditions as above (Table 1).

For each KI peak, the mean and $95 \%$ confidence interval (CON) for observations around the mean were computed for each species (60 Asian and 60 German cockroaches, stages pooled within species); 12 common peaks (see Table 2) had nonoverlapping intervals for the two species, and these were selected for further analysis. These data were augmented by an additional 30 German cockroaches (described above) and data from all 150 specimens were subjected to principal components analysis (SAS Institute 1985) to visualize clustering of specimens by species and to assess uniformity (i.e., sexual dimorphism) within each species. Kaiser's measure of sampling adequacy (Kaiser 1970, Cerny \& Kaiser 1977) was used to confirm the suitability of each peak for aiding in the identification of each species.

These 12 peaks were used to develop a scoring procedure for identification of 150 individual specimens. Each was given a score of 1 or -1 for each of the 12 peaks if the value was within the range of Asian or German CON, respectively, or a score of 0 if it fell outside of both ranges. All 12 scores were totaled for each specimen; possible scores therefore ranged from 12 to -12 . Mean cumulative discrimination scores then were computed by life stage $(n=10$, except for Alaskan German cockroaches where $n=5$ ), and $99 \%$ CON for observations about the means were constructed and plotted. Critical regions were then chosen for each species, based on the range of these $99 \% \mathrm{CON}$. 
Table 1. Alkanes and methylalkanes of German (HRDC and New Caledonia [NCal.] strains) and Asian cockroach females identified by mass spectrometry

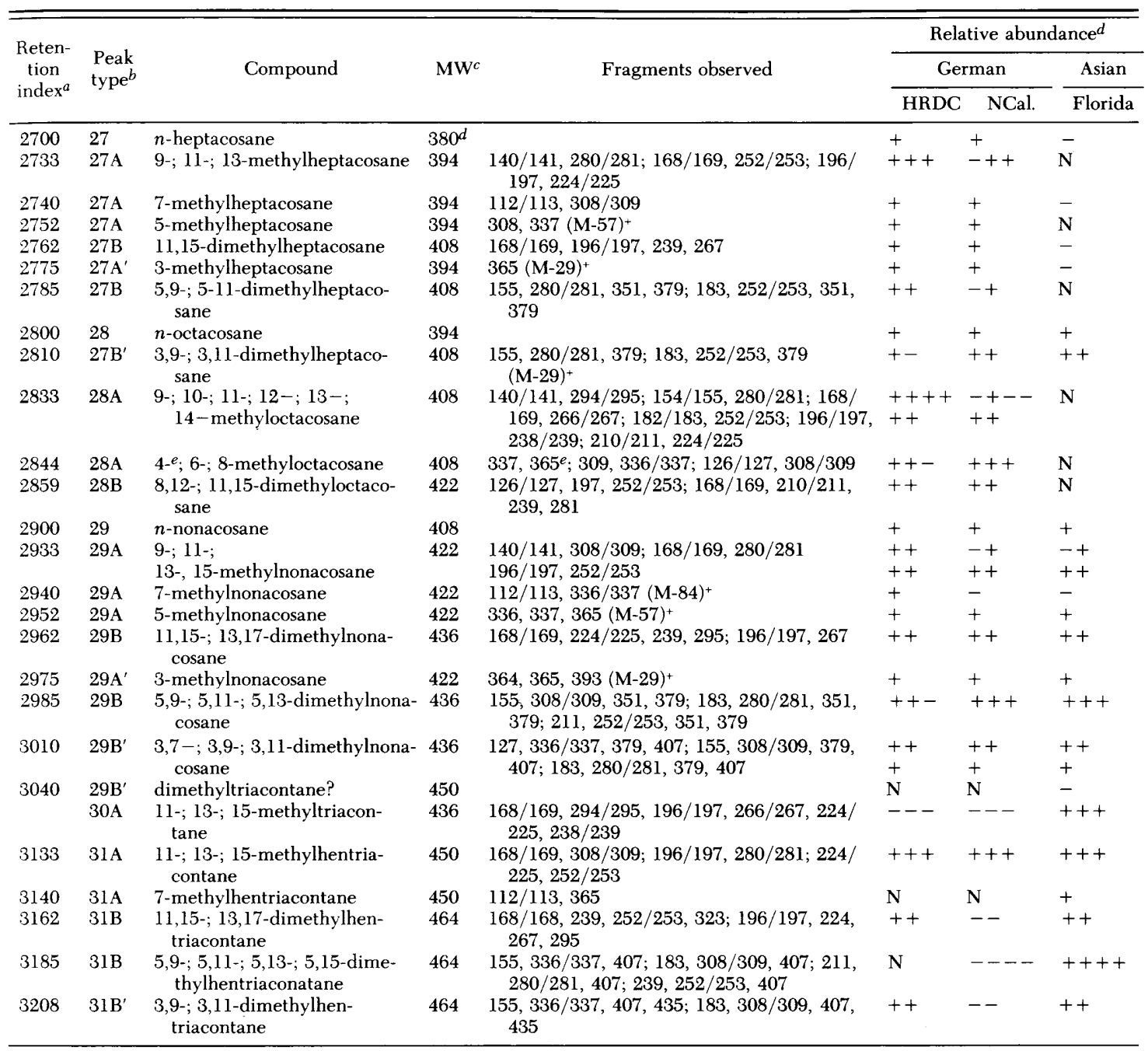

a Nonpolar capillary $30 \mathrm{~m} \times 0.32 \mathrm{~m}$ ID DB-1.

${ }^{b}$ A, methyl; A', 2-, 3-, or 4-methyl; B, dimethyl; B', 2,X- or 3,X-dimethyl (Nelson \& Sukkestad 1970).

${ }^{c}$ Molecular weight derived from $(\mathrm{M}-1)^{+}$via chemical ionization (methane) GCMS

$d+$, present; - , not observed; $\mathbf{N}$, structure not determined but $(\mathbf{M}-1)^{+}$confirmed by chemical ionization chromatography.

e 4-methyloctacosane eluted at KI 2859.

These discrimination scores, computed for each specimen for each KI and totaled to produce a cumulative score per specimen, were normalized by ranking all observations by species (Conover \& Iman 1981), and ranks were subjected to analysis of variance (GLM procedure, SAS Institute 1985) to assess heterogeneity of cumulative scores within each species.

A single blind test was used from different populations to verify reliability of this identification procedure. Asian cockroaches were field-collected in rural areas east of Tampa; German cockroaches were taken from museum specimens (10) collected in New Caledonia in 1961 , and from a colony $\left(\mathbf{F}_{2}\right.$ generation) that originated from specimens collected at an infested house near Lakeland, Fla., and from an apartment complex in Gainesville, Fla. Cockroach body parts (legs, wings, cast skin), as well as whole bodies and oöthecae were tested; 12 hybrids were also included from German females (HRDC strain). Identifications were first made from visual examination of the chromatograms. Data from the $12 \mathrm{KI}$ peaks were then used to score each "unknown," and cumulative scores for each specimen were compared to established critical score intervals to categorize each as Asian, 
Table 2. Percentages of total composition and ranges of hydrocarbons of adult German (HRDC strain) and Asian cockroaches by gas chromatography

\begin{tabular}{|c|c|c|c|c|c|c|c|c|}
\hline $\begin{array}{l}\text { GC peaks } \\
\quad(\mathrm{KI})^{a}\end{array}$ & \multicolumn{4}{|c|}{ Asian $(n=60)$} & \multicolumn{4}{|c|}{ German $(n=60)$} \\
\hline $2700^{*}$ & 0.03 & 0.02 & 0.01 & 0.08 & 1.60 & 0.29 & 1.01 & 2.29 \\
\hline $2753^{*}$ & 0.04 & 0.02 & 0.01 & 0.08 & 2.14 & 0.56 & 0.60 & 3.37 \\
\hline $2775-2785^{*}$ & 0.08 & 0.03 & 0.03 & 0.16 & 5.87 & 0.86 & 3.21 & 7.86 \\
\hline $2810^{*}$ & 0.94 & 0.78 & 0.12 & 3.79 & 5.71 & 1.50 & 3.43 & 10.66 \\
\hline 2875 & 0.40 & 0.14 & 0.01 & 0.94 & 0.87 & 0.19 & 0.54 & 1.34 \\
\hline 2900 & 6.16 & 1.40 & 3.60 & 9.27 & 6.79 & 1.36 & 4.21 & 9.78 \\
\hline 2933 & 8.99 & 3.16 & 6.02 & 18.73 & 16.36 & 3.20 & 12.53 & 26.30 \\
\hline $2952-2962$ & 12.48 & 1.81 & 9.60 & 19.29 & 11.45 & 0.99 & 9.23 & 13.27 \\
\hline $2975-2985$ & 15.29 & 1.48 & 11.35 & 18.19 & 13.65 & 1.82 & 9.49 & 17.40 \\
\hline 3010 & 24.21 & 3.72 & 16.32 & 30.74 & 15.26 & 1.97 & 9.82 & 18.82 \\
\hline 3152 & 0.58 & 0.56 & 0.01 & 2.48 & 0.50 & 0.18 & 0.01 & 0.93 \\
\hline $3185^{*}$ & 3.41 & 0.67 & 1.91 & 4.95 & 0.32 & 0.09 & 0.11 & 0.52 \\
\hline $3208^{*}$ & 2.91 & 0.80 & 1.85 & 6.49 & 0.34 & 0.13 & 0.01 & 0.67 \\
\hline 3233 & 0.61 & 0.26 & 0.23 & 1.48 & 0.00 & 0.00 & 0.00 & 0.00 \\
\hline
\end{tabular}

${ }^{a} \mathrm{KI}$ values followed by an asterisk are those with $95 \% \mathrm{CON}$ that do not overlap between species; these were used in the principal components analysis.

German, or a hybrid cockroach. Voucher Asian and German cockroaches were deposited at the U.S. National Museum of Natural History, Smithsonian Institution, Washington, D.C.

\section{Results}

The patterns of hydrocarbon components of males, females, and nymphs within each species were consistent. German cockroaches had a cluster of medium-sized peaks eluting after heptacosane (KI 2700-2810), a cluster of much larger peaks that eluted after nonacosane (KI 2900-3040), relatively tiny peaks that eluted after hentriacontane (KI $3100-3230$ ), and virtually no peaks that eluted after tritriacontane (33 carbons).

All chromatograms display the 10-20 min portion of each GC run (Fig. 1). No appreciable differences were seen in patterns of individuals from the populations examined, including HRDC, Orlando normal, Alaska colony, Lakeland wild, New Caledonia museum, and specimens submitted from throughout the Gulf states. Most material (approximately $91 \%$ ) was seen in 14 peaks that eluted at KI 2700, 2733, 2752, 2775, 2785, 2810, 2900, 2933, 2952, 2962, 2975, 2985, 3010, and 3133 .

In contrast, Asian specimens had very little hydrocarbon that eluted before nonacosane (KI 2700 2833), a cluster of large peaks that eluted after nonacosane (KI 2900-3040), a medium-sized cluster of peaks that eluted after hentriacontane (KI 3100-3230), and again, virtually no peaks that eluted after tritriacontane (33 carbons) (Fig. 2). Most material (approximately 93\%) was seen in 11 peaks that eluted at KI 2900, 2933, 2952, 2975, 2985, $3010,3040,3100,3133,3185$, and 3208 .
The methyl alkanes of German and Asian females are described here in groups by the GC peak in which they eluted, and by their molecular weights, as determined from CI spectra (Table 1). Alkane retention times were consistent with standards; a few alkenes were found but not characterized. The $27 \mathrm{~A}$ and $27 \mathrm{~B}$ structures were determined only in German females; the former (KI 2733) included 13-, 9-, and 11-methyl isomers in decreasing order of intensity, and the KI 2740 peak contained the 7 -isomer. The 29A peak (KI 2933) was similarly a mixture, with 13-, 15-, and 11methyl isomers present in both species, but the 9-methyl was less evident in Asian specimens. The 27A (KI 2752) and 29A peaks (KI 2952) contained only 5-methyl alkanes. The $27 \mathrm{~A}^{\prime}$ (KI 2775) and $29 \mathrm{~A}^{\prime}$ (KI 2975) peaks contained 3-methyl alkanes having prominent M-29 fragment ions, consistent with Nelson and Sukkestad (1970). A 4-methyl alkane would have eluted just before a 3-methyl alkane, but it was observed only in the KI 2859 peak in both German cockroach samples. The 31A peak (KI 3133) was large in Asian females and contained 11-, 15-, and 13-methylhentriacontanes.

The earliest eluting dimethyl alkanes in each carbon series were those with internal 3-methylene bridges, observed at KI 2762, KI 2859, and KI 2962 in German cockroaches, with KI 3162 also present in Asian cockroaches. The spectra were consistent with the 13,17-, and 11,15-dimethyl structures proposed. The next eluting 5,X-dimethyl alkanes had methyl branches closer to the end of the chain, contained 3-, 5-, and 9-methylene bridges, and eluted at KI 2785, KI 2885, and KI 2985. The 5,9-, 5,11-, and 5,13-dimethyl structures proposed were consistent with the spectra. These structures were 


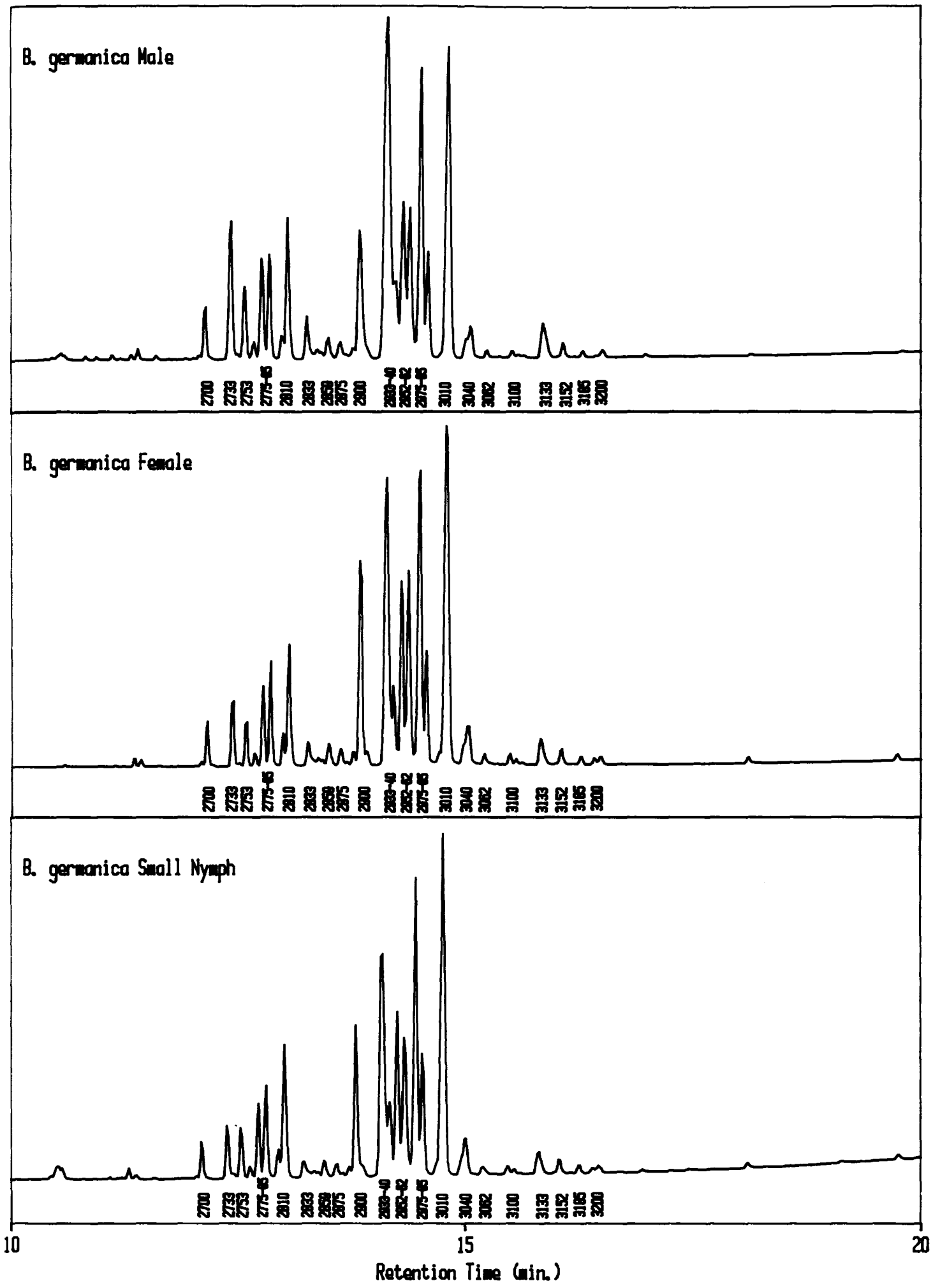

Fig. 1. Gas chromatograms of cuticular hydrocarbons of the German cockroach, B. germanica (HRDC strain), characteristic for males, females, and nymphs. 


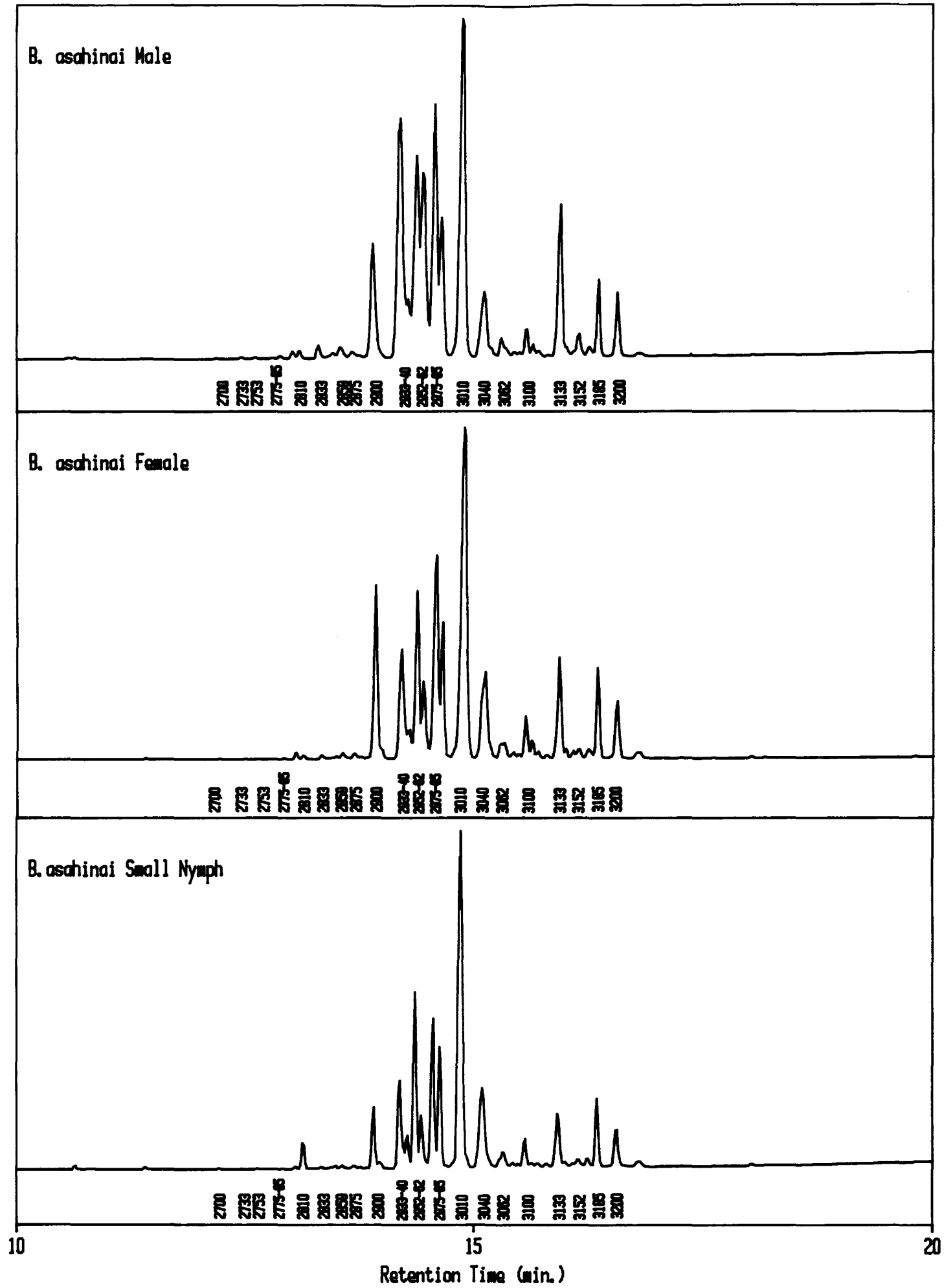

Fig. 2. Gas chromatograms of cuticular hydrocarbons of the Asian cockroach, B. asahinai (Lakeland, Fla.), characteristic for males, females, and nymphs. 
determined for KI 2785 only in German cockroaches; a 5,15-dimethyl homolog was present in Asian cockroaches at KI 3185. The next eluting dimethyl alkanes at KI $2810\left(27 \mathrm{~B}^{\prime}\right)$ and KI 3010 $\left(29 \mathrm{~B}^{\prime}\right)$, were homologous in structure in both species. Spectra were consistent with 3,11-dimethyl alkanes, as the consistently large KI 3010 peak showed major fragments at $\mathrm{m} / \mathrm{z} 182 / 183,280$, and 407 (loss of $2 \mathrm{C}$ ), and were consistent with published spectra for the synthesized compound, 3,11-dimethylnonacosane (Pomonis et al. 1980). This peak also contained minor amounts of 3,9- and 3,7-homologs in both species, whereas a KI 3208 peak in Asian cockroaches had 3,9- and 3,11-dimethyl alkane homologs. The mass spectra of hydrocarbons from male German and Asian cockroaches were obtained; the components were entirely consistent with structures of compounds reported here for conspecific females.

Only when compounds had even-numbered backbones, as at KI 2833 and KI 2859, were methyl branches observed at even-numbered carbons; this made them more difficult to identify in the presence of compounds with methyl branches on oddnumbered carbons. Also, the KI 3040 peak contained an undetermined dimethyl alkane with some monomethyl alkanes in German cockroaches, but it was not present in Asian samples according to the CI ion chromatograms. This data-handling feature was helpful in describing coeluting compounds that differ by one carbon.

For B. vaga, only higher molecular weight materials with hydrocarbons of 31-44 carbons (KI 3162-4410) were present consistently (Fig. 3). Most material was seen in nine peaks that eluted at KI $3162,3175,3800,3810$ (doublet), 4000, 4010 (doublet), 4200, 4210 (doublet), and 4400, 4410 (doublet).

A few minor components, common to Asian and German specimens, were observed by GC $(\bar{x} \%$ composition from three males), including KI 2700 $(0.02 \%), 2810(0.13 \%), 2900(0.07 \%), 3062$ (0.48\%), and $3100(0.43 \%)$. Other major components not found in Asian or German cockroaches included KI $3165(6.9 \%), 3175(1.9 \%), 3210(0.8 \%), 3265$ $(0.8 \%), 3275(0.8 \%), 3365(0.6 \%), 3375(0.8 \%), 3410$ $(4.7 \%), 3800(4.5 \%), 3810(17.6 \%), 4000(14.9 \%)$, 4010 (17.5\%), 4200 (7.8\%), $4210(4.4 \%), 4400(1.0 \%)$, and $4410(0.8 \%$, total $87 \%)$.

Compositions were consistent among males, females, and nymphs from colony material, as well as from museum materials collected in several western states. Mass spectra of the major peaks indicated methyl alkanes at KI $3162(\mathrm{~m} / \mathrm{z} 407$ [22\%], 435 [5\%], and 449 [ $100 \%$ by CI, M-1]) for 2-methylhentriacontane, KI $3175(\mathrm{~m} / \mathrm{z} 393$ [12\%], 421 [12\%], and 449 [100\% by CI, M-1]) for 3-methylhentriacontane. The KI 3210 peak contained a mixture of undetermined methyl alkanes $(\mathrm{m} / \mathrm{z} 449$ and 463 [100\% by CI, M-1]), but 3410 contained a mixture of undetermined dimethyl alkanes $(\mathrm{m} / \mathrm{z} 491$ [100\% by CI, M-1]). An unusual series of alkatrienes and another of dienes were observed as pairs of peaks, with trienes eluting first. Complete structural identification of the mixtures of trienes and dienes is beyond the scope of this paper. However, the $\mathrm{CI}$ spectra clearly showed M-1 base peaks as follows: KI 3800 (C39:3, m/z 541), 3810 (C39:2, $\mathrm{m} / \mathrm{z} 543$ ), 4000 (C41:3, m/z 569), 4010 (C41:2, $\mathrm{m} / \mathrm{z} 571)$, and $4200(\mathrm{C} 43: 3, \mathrm{~m} / \mathrm{z} 597)$. The KI 4210 peak and those that eluted later were not recorded.

The comparison of GC data among 60 Asian and 60 German specimens (HRDC strain) indicated that 26 peaks were present consistently, regardless of age or sex, and the percentage compositions of 21 peaks were compared (Table 2). Except for KI 3152 , there were statistically significant differences between species $(P<0.01$, Duncan-Waller test, SAS Institute 1985). Although this analysis is clearly sufficient to separate populations of each species, it may be inadequate for identifying individual specimens. Consequently, salient differences among peaks were sought that could be used to identify individual specimens. Therefore, the 12 peaks (see Table 2) for which the 95\% CON for Asian and German cockroaches did not overlap were selected for further scrutiny. Kaiser's measure of sampling adequacy (MSA) confirmed that scores for these peaks were well above the level of minimal acceptance (0.8, SAS Institute 1985), ranging from 0.916 (3185) to 0.978 (3100), indicating that each aided in separating species. Consequently, these data were retained for computing first and second principal components.

All specimens (150) were plotted by first (ordinate) and second (abscissa) principal components to visualize how well the combination of the 12 peaks separates species, and to assess uniformity within species (Fig. 4A). The species were widely separated by first component scores.

Examination of scores for adults indicated heterogeneity within species (Fig 4B). Male Asian cockroaches $(\mathrm{m})$ scored lower than females (f) for both components, indicating sexual dimorphism of the species for extracted hydrocarbons. Among German cockroaches, those affected by hydroprene $(\mathrm{H})$ were characterized by relatively high first component scores and relatively low second component scores. Specimens from the Alaska colony $(K)$ also were clustered, but scored higher than hydropreneaffected German cockroaches for both components. As in Asian cockroaches, occurrence of sexual dimorphism in German cockroaches was illustrated by the separate clustering of males from females (M and F). Specimens from the Gulf states (S) were scattered among the other groups, indicating the greatest heterogeneity among sample groups of German cockroaches.

Within each species, mean discrimination scores varied significantly among stages, corroborating the evidence for heterogeneity indicated in principal components analysis (Table 3). Overall means were close to the maximum possible scores of 12 or -12 . Overall mean cumulative scores were 11.05 and 


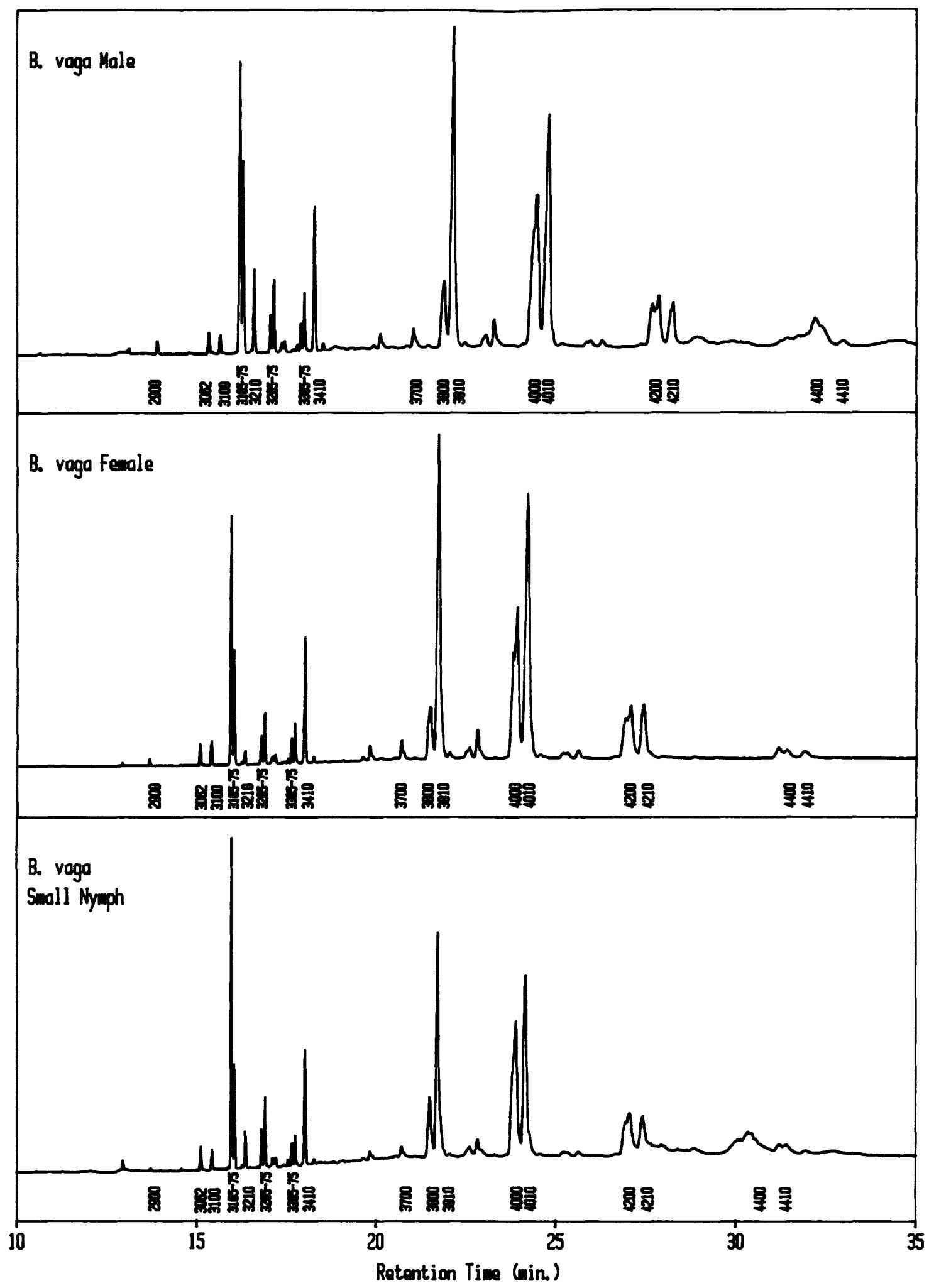

Fig. 3. Gas chromatograms of cuticular hydrocarbons of the field cockroach, B. vaga (colony), characteristic for males, females, and nymphs. 

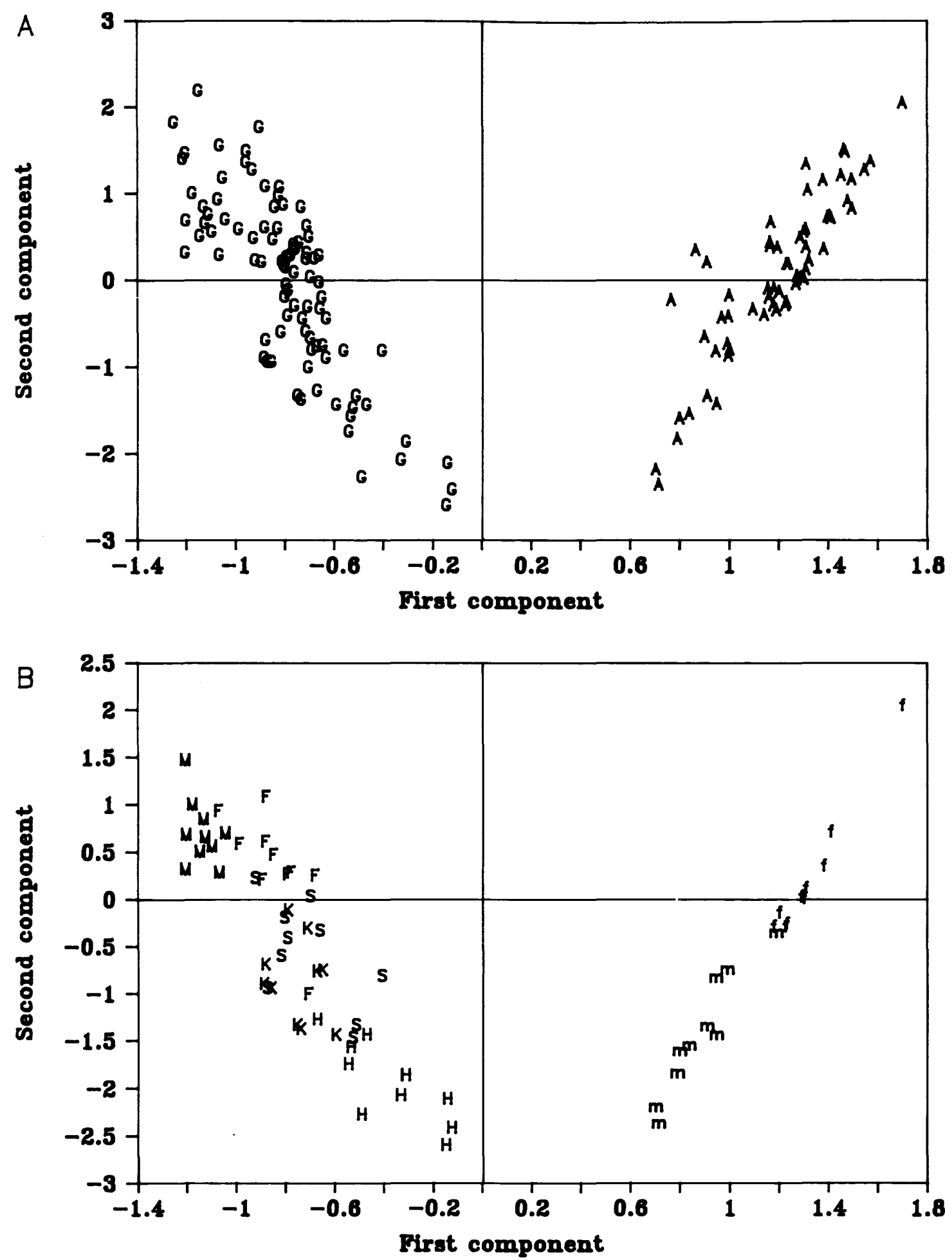

Fig. 4. (A) Separation of Asian (A) and German (G) cockroach specimens based on first and second principal components analysis of cuticular hydrocarbon data; (B) separation of adults by sex or strain; M, German male; F, German female; S, German cockroaches from the Gulf states; K, Alaska colony; H, hydroprene-affected adults; $\mathrm{m}$, Asian male; $f$, Asian female. 


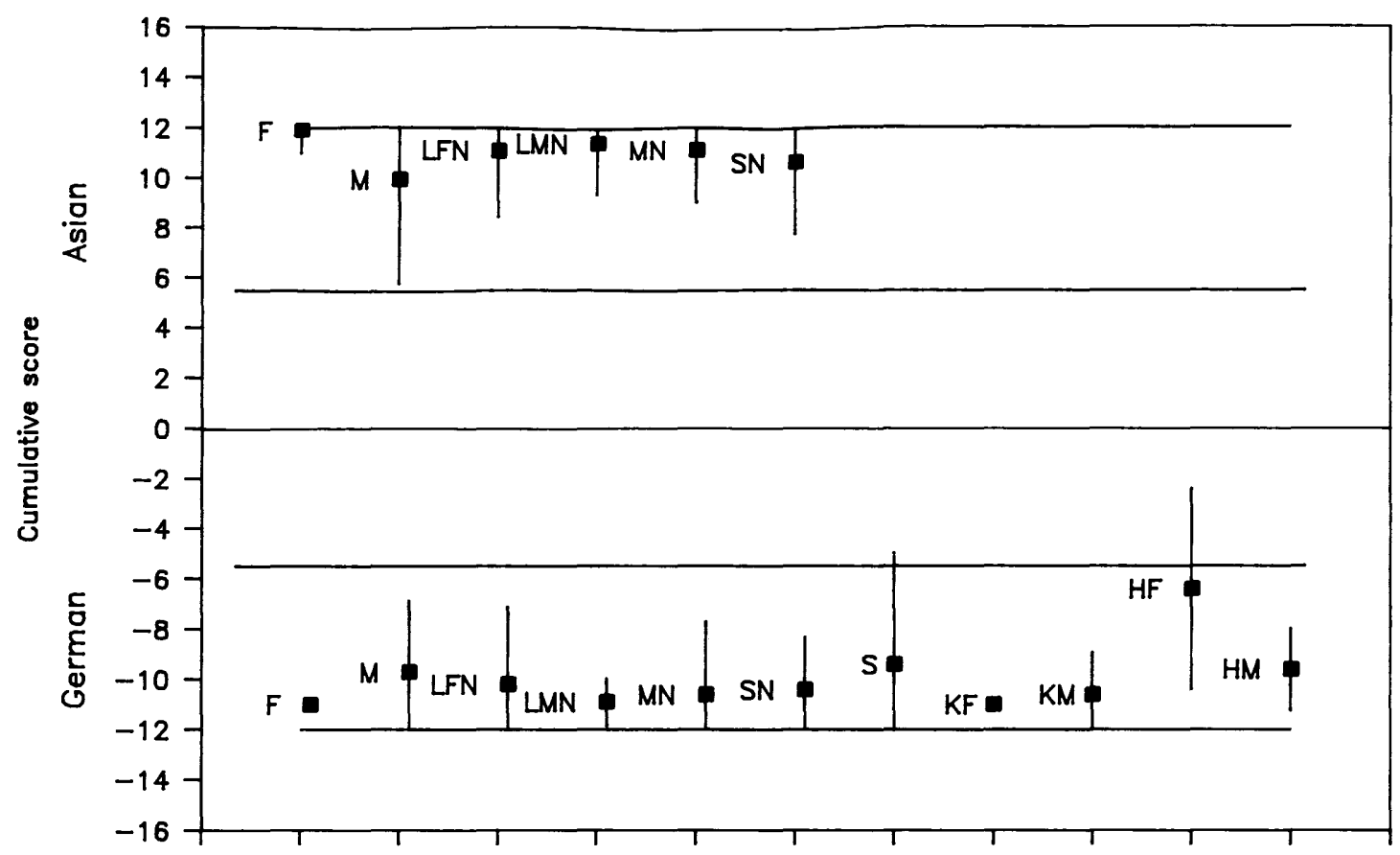

Species and stage

Fig. 5. Mean cumulative scores ( $)$ and $99 \% \mathrm{CON}$ (vertical bars) of observations for various stages or strains of German and Asian cockroaches. All positive values are Asian cockroaches, and all negative values are German cockroaches; F, female; M, male; LFN, large female nymph; LMN, large male nymph; MN, medium nymph; SM, small nymph; S, German cockroaches (wild) from the Gulf states; KF, Alaskan colony females; KM, Alaskan colony males; HF, hydroprene-affected females; and HM, hydroprene-affected males. Horizontal lines indicate arbitrarily selected limits for critical diagnostic values (Asian $=5.5,12$; German $=-12,-5.5)$.

Table 3. Ranges and means of cumulative scores for life stages of Asian and German cockroaches

\begin{tabular}{|c|c|c|c|c|c|c|c|}
\hline Species & Stage $^{a}$ & $n$ & Min & $\operatorname{Max}$ & $\mathrm{SD}$ & SEM & $\operatorname{Mean}^{b}$ \\
\hline Asian & $\begin{array}{l}\text { F } \\
\text { M } \\
\text { LFN } \\
\text { LMN } \\
\text { MN } \\
\text { SN } \\
\text { Total }\end{array}$ & $\begin{array}{l}10 \\
10 \\
10 \\
10 \\
10 \\
10 \\
60\end{array}$ & $\begin{array}{r}11 \\
7 \\
10 \\
10 \\
10 \\
9 \\
7\end{array}$ & $\begin{array}{l}12 \\
12 \\
12 \\
12 \\
12 \\
12 \\
12\end{array}$ & $\begin{array}{l}0.31 \\
1.37 \\
0.88 \\
0.70 \\
0.70 \\
0.97 \\
1.06\end{array}$ & $\begin{array}{l}0.10 \\
0.43 \\
0.28 \\
0.22 \\
0.22 \\
0.31 \\
0.14\end{array}$ & $\begin{array}{c}11.9 \mathrm{a} \\
9.9 \mathrm{~d} \\
11.1 \mathrm{bc} \\
11.4 \mathrm{ab} \\
11.4 \mathrm{ab} \\
10.6 \mathrm{~cd} \\
11.1\end{array}$ \\
\hline $\begin{array}{l}\text { German } \\
\quad(\text { HRDC })\end{array}$ & $\begin{array}{l}\text { F } \\
\text { M } \\
\text { LFN } \\
\text { LMN } \\
\text { MN } \\
\text { SN }\end{array}$ & $\begin{array}{l}10 \\
10 \\
10 \\
10 \\
10 \\
10\end{array}$ & $\begin{array}{l}-11 \\
-11 \\
-11 \\
-11 \\
-11 \\
-11\end{array}$ & $\begin{array}{r}-11 \\
-8 \\
-8 \\
-10 \\
-8 \\
-9\end{array}$ & $\begin{array}{l}0.00 \\
0.95 \\
1.03 \\
0.32 \\
0.97 \\
0.70\end{array}$ & $\begin{array}{l}0.00 \\
0.30 \\
0.33 \\
0.10 \\
0.31 \\
0.22\end{array}$ & $\begin{array}{c}-11.0 \mathrm{~g} \\
-9.7 \mathrm{bcd} \\
-10.2 \mathrm{cde} \\
-10.9 \mathrm{cde} \\
-10.6 \mathrm{efg} \\
-10.4 \mathrm{def}\end{array}$ \\
\hline $\begin{array}{l}\text { German } \\
\text { (Orlando-hydroprene) } \\
\text { German } \\
\text { (Alaska) } \\
\text { German }\end{array}$ & $\begin{array}{l}\mathrm{F} \\
\mathrm{M} \\
\mathrm{F} \\
\mathrm{M} \\
\mathrm{A}\end{array}$ & $\begin{array}{r}5 \\
5 \\
5 \\
5 \\
10\end{array}$ & $\begin{array}{r}-8 \\
-10 \\
-11 \\
-11 \\
-11\end{array}$ & $\begin{array}{r}-5 \\
-9 \\
-11 \\
-10 \\
-7\end{array}$ & $\begin{array}{l}1.34 \\
0.55 \\
0.00 \\
0.55 \\
1.43\end{array}$ & $\begin{array}{l}0.60 \\
0.24 \\
0.00 \\
0.25 \\
0.45\end{array}$ & $\begin{array}{l}-6.4 \mathrm{a} \\
-9.7 \mathrm{~b} \\
-11.0 \mathrm{~g} \\
-10.6 \mathrm{efg} \\
-9.4 \mathrm{bc}\end{array}$ \\
\hline & Total & 90 & -11 & -5 & 1.34 & 0.14 & -10.1 \\
\hline
\end{tabular}

${ }^{a} \mathrm{~A}$, adults; $\mathrm{F}$, female; M, male; LFN, large female nymph; LMN, large male nymph; $\mathrm{MN}$, medium nymph; SN, small nymph.

${ }^{b}$ Means of stages in the same species followed by the same letter are not significantly different $(P<0.05$; Waller-Duncan multiple range test [SAS Institute 1985] applied to ranked observations [Conover \& Iman 1981]). 


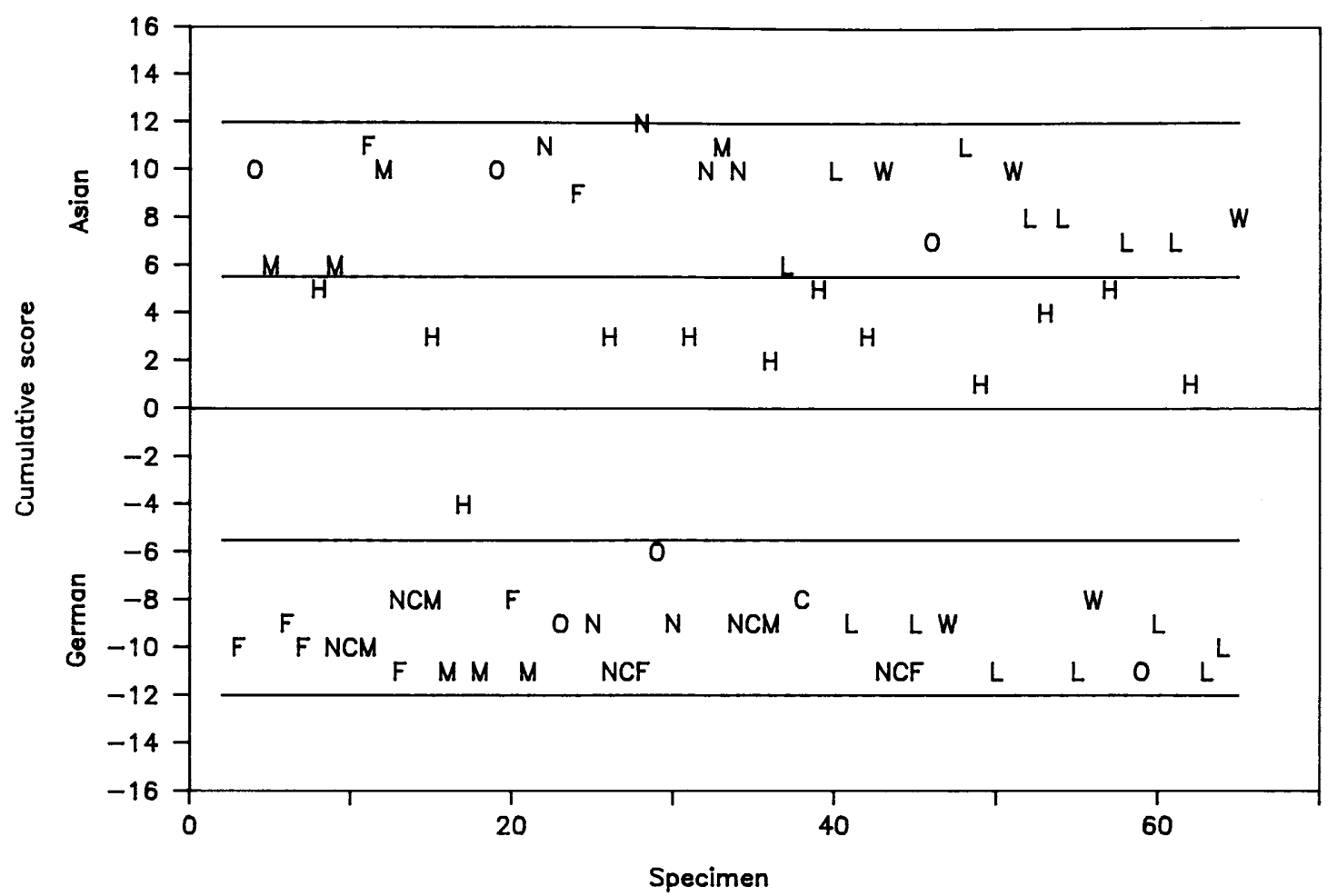

Fig. 6. Plot of cumulative scores for 63 specimens submitted as a blind test for evaluating the reliablility of critical diagnostic values (see Fig. 5) for separating Asian and German cockroaches; C, cast skin; F, female; H, hybrid; L, leg; M, male; N, nymph; NCF, New Caledonia female (German); NCM, New Caledonia male (German); $\mathrm{O}$, oötheca; and W, wing. Limits for critical diagnostic values are represented by horizontal lines (see text).

-10.1 for Asian and German cockroaches, respectively, indicating that the combination of these 12 KI peaks clearly is diagnostic for each species. Therefore, mean cumulative scores and $99 \%$ CON (three standard deviations) of observations about the mean were plotted (Fig. 5) for each group listed in Table 3. Among Asian cockroaches, values were lowest and most variable for males and small nymphs. Consequently, in determining a range of critical cumulative scores that would be useful for all stages of Asian cockroaches, we arbitrarily reduced the computed lower limit from 7.87 to 5.5 to encompass the anticipated variability of males. Similarly, among German cockroaches, males and specimens from the Gulf states (Fig. 5, S) were lowest and most variable of those stages not affected by hydroprene; the computed upper limit for critical cumulative scores of -6.09 was adjusted to -5.5 .

The reliablility of this was assessed by submitting 63 specimens for single-blind testing. In all cases, visual inspection of the chromatograms gave correct identifications of 10 Asian and 10 German cockroaches, 5 German cockroaches from New Caledonia (museum specimens collected in the 1960s), three oöthecae each from Asian and German cockroaches, seven legs from seven specimens each of Asian and German cockroaches, wings from
3 Asian and 2 German cockroaches, and a cast skin from a nymphal German cockroach; 12 hybrids from Asian females were also correctly identified. Plotting cumulative scores computed from the 12 KI criteria (Fig. 6) also resulted in correct identification of all 23 Asian and 28 German samples. Additionally, all 12 hybrids scored between Asian and German ranges (Fig. 6), and each chromatogram was characteristic of hybrids (Fig. 7).

\section{Discussion}

Use of these identification methods may be useful in complementing or supplanting traditional taxonomy methods based on morphological differences. As mentioned previously, identification of male German and Asian cockroaches can be done morphologically, but there are few identifying characters for females (Mizukubo 1981), and virtually none for nymphs (Roth 1985). Identifications based on dissections are not easy at a field location or by pest control operators. Although only Asian cockroach adults fly, this diagnostic behavior might not be observed indoors, or at times when adults are rare relative to nymphs. Consequently, identifying infestations under these circumstances, based solely on established morphological characteristics, is virtually impossible. Moreover, hydroprene, 


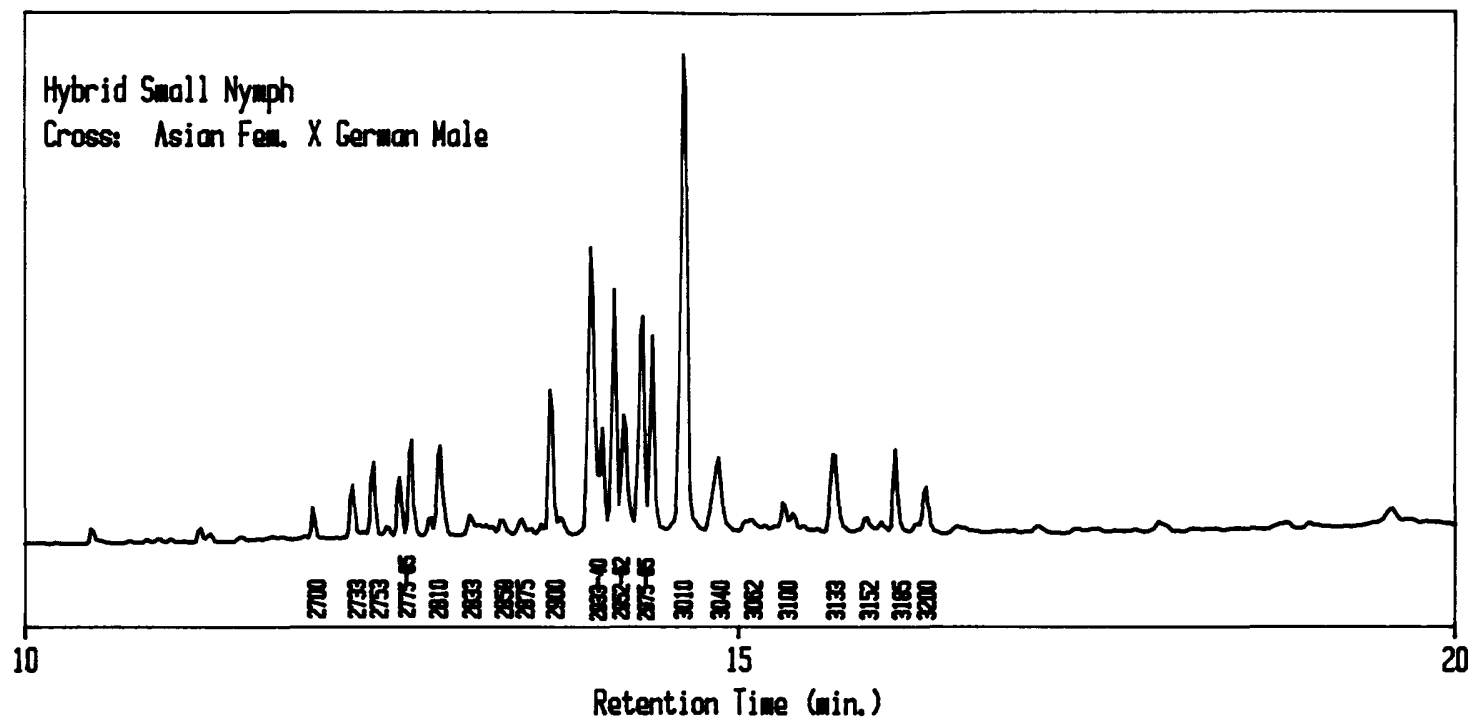

Fig. 7. Gas chromatogram of hydrocarbons from a German-Asian hybrid small nymph.

which is a highly effective chemical used to control the German cockroach, causes twisted, distorted wings in the adults of the German (Patterson \& Koehler 1985) and the Asian (R. S. Patterson, personal communication) cockroaches. This situation negates the use of wing measurements or flight ability to identify which species constituted an infestation.

The hydrocarbon components of each Blattella species were clearly different by simple inspection of chromatograms, regardless of age, sex, or geographical origin. For example, chromatographic patterns of museum specimens of German cockroaches from New Caledonia (collected in 1961) were similar to those of all other German specimens from Alaska, colonies obtained from field collections in Washington, D.C. (HRDC strain), and from Florida and other Gulf states. Consequently, it appears that the phenomenon of hydrocarbon-pattern specificity for the species tested here is universal.

Sticky traps are used commonly in assessing cockroach population structure. Single legs or wings give diagnostic chromatograms; body parts, uncontaminated or slightly contaminated by trap adhesives, can be removed for analysis from specimens that appear morphologically to be either German or Asian. This procedure also would let researchers retain live specimens (i.e., hybrids) and still determine lineage.

The chromatograms shown here are consistent with early GC studies of cockroaches. Bennett \& Hall (1973) pyrolyzed 2-mg samples of dried $B$. germanica exoskeletal body parts, and observed hydrocarbons similar to those reported here, though poorly resolved, and contaminated by nonhydrocarbons. Acree et al. (1965) analyzed crude hemolymph supernate of several species of male cockroaches and found significant differences among five species in unidentified hydrocarbons. Although no Asian cockroaches or B. vaga were investigated in that study, chromatograms of male German cockroaches were similar to the patterns seen here.

Mass spectrometry confirmed the assignments of retention indexes for the species studied here. These experiments lend confidence in establishing the utility of gas chromatography for identification of closely related species of insects where traditional morphology-based techniques are difficult or not diagnostic for all stages. This technique allows rapid and confident scoring of unknown specimens that can be compared to scores of known specimens.

\section{Note added in proof:}

After submission of this manuscript, the GCMS of hydrocarbons of one sample of mixed-sex $B$. germanica from Europe were published, with the same hydrocarbon pattern for this species as described here. They identified fewer compounds, and did not identify branching patterns in dimethylheptacosane and dimethylnonacosane, but the major peaks included the same compounds as reported in this work (Augustynowicz et al. 1987).

\section{Acknowledgment}

We thank J. Hector for his excellent technical assistance in chromatography and data preparation. Thanks to L. Roth and T. Mizukubo for identifying the $B$. asahinai and to the latter for helpful comments on the manuscript. Special thanks go to $M$. Rust, University of California, Riverside, for the starter colony of $B$. vaga. 


\section{References Cited}

Acree, F., Jr., R. B. Turner, B. J. Smittle \& G. S. Burden. 1965. Hydrocarbons in haemolymph of cockroaches of different ages. J. Insect Physiol. 11: 905-910.

Augustynowicz, M., E. Malinski, Z. Warnke, J. Szafranek \& J. Nawrot. 1987. Cuticular hydrocarbons of the German cockroach, Blattella germanica $\mathrm{L}$. Comp. Biochem. Physiol. 86B: 519-523.

Bartelt, R. J., M. T. Armold, A. M. Schaner \& L. L. Jackson. 1986. Comparative analysis of cuticular hydrocarbons in the Drosophila virilis species group. Comp. Biochem. Physiol. 83B: 731-742.

Bennett, G. W. \& R. C. Hall. 1973. Identification of cockroach exoskeletal fragments using pyrolysis-gas chromatography. Ann. Entomol. Soc. Am. 66: 11231127.

Brenner, R. J., R. S. Patterson \& P. G. Koehler. 1988. Ecology, behavior, and distribution of Blattella asahinai (Orthoptera; Blattaria) in Central Florida. Ann. Entomol. Soc. Am. 81: 432-436.

Carlson, D. A. \& A. B. Bolten. 1984. Identification of Africanized and European honey bees, using extracted hydrocarbons. Bull. Entomol. Soc. Am. 30 $32-35$.

Carlson, D. A. \& M. W. Service. 1980. Identification of mosquitoes of Anopheles gambiae species complex A \& B by analysis of cuticular components. Science 207: 1089-1091.

Castner, J. L. \& J. L. Nation. 1984. Cuticular lipids for species recognition of mole crickets (Orthoptera: Gryllotalpidae) I. Scapteriscus didactylus, Scapteriscus imitatus, and Scapteriscus vicinus. Fla. Entomol. 67: $155-160$

Cerney, B. A. \& H. F. Kaiser. 1977. A study of a measure of sampling adequacy for factor-analytic correlation matrices. Multivar. Behav. Res. 12: 289325 .
Conover, W. J. \& R. L. Iman. 1981. Rank transformations as a bridge between parametric and nonparametric statistics. Am. Stat. 35: 124-129.

Howard, R. W. 1987. Chemosystematic studies of the Triboliini (Coleoptera: Tenebrionidae): phylogenetic inferences from the defensive chemical of eight $\mathbf{T r}$ bolium spp., Palorus ratzeburgi (Wissman), and Latheticus oryzae (Waterhouse). Ann. Entomol. Soc. Am. 80: 397-405.

Kaiser, H. F. 1970. A second generation little jiffy. Psychometrika 35: 401-415.

Mizukubo, T. 1981. A revision of the genus Blattella (Blattaria: Blattellidae) of Japan. I. Terminology of the male genitalia and description of a new species from Okinawa Island. Esakia 17: 149-159.

Nelson, D. R. \& D. R. Sukkestad. 1970. Normal and branched hydrocarbons from eggs of the tobacco hornworm. Biochemistry 9: 4601-4611.

Patterson, R. S. \& P. G. Koehler. 1985. Sterility: a practical IPM approach for German cockroach (Blattella germanica) control. Proceedings, 1st Insect Growth Regulator Symposium 1(1): 48-60.

Pomonis, J. G., D. R. Nelson \& C. L. Fatland. 1980. Insect hydrocarbons 2. Mass spectra of dimethylalkanes and the effect of the number of methylene units between methyl groups on fragmentation. J. Chem. Ecol. 6: 965-972.

Roth, L. W. 1985. A taxonomic revision of the Genus Blattella Caudell (Dictyoptera, Blattaria; Blattellidae). Entomol. Scand. Suppl. 22.

1986. Blattella asahinai introduced into Florida (Blattaria; Blattellidae). Psyche 93: 371-374.

SAS Institute. 1985. SAS/STAT guide for personal computers, version 6 ed. SAS Institute, Cary, N.C.

Received for publication 31 July 1987; accepted 9 May 1988. 